\title{
Radio source structure and VLBI position instabilities
}

\author{
Romuald Bouffet* \\ Univ. Bordeaux, LAB, UMR 5804, F-33270, Floirac, France. \\ CNRS, LAB, UMR 5804, F-33270, Floirac, France \\ E-mail: bouffet@obs.u-bordeauxl. fr

\section{Patrick Charlot} \\ Univ. Bordeaux, LAB, UMR 5804, F-33270, Floirac, France. \\ CNRS, LAB, UMR 5804, F-33270, Floirac, France \\ E-mail: charlot@obs.u-bordeauxl. fr
}

\section{Sébastien Lambert}

Observatoire de Paris - SYRTE, CNRS/UMR 8630 \& Université Pierre et Marie Curie, 75005

Paris, France

E-mail: Sebastien. Lamberteobspm.fr

\begin{abstract}
Astrometric positions of extragalactic radio sources derived from VLBI data are used to build highly-accurate reference frames such as the International Celestial Reference Frame. Despite their distant locations, instabilities in the position of these sources are often seen on time scales of months to years, which is generally thought to be caused by source structure evolution. In this paper, we compare position instabilities and structural evolution for a sample of 68 sources observed over a 10-year period (1994-2003). Our results indicate that the two phenomena are linked at some level although the correlation is not perfect.
\end{abstract}

11th European VLBI Network Symposium \& Users Meeting

9-12 October 2012

Bordeaux (France)

\footnotetext{
*Speaker.
} 


\section{Introduction}

The current IAU fundamental celestial reference frame, namely the ICRF2 (second realization of the International Celestial Reference Frame), which has been in use since January 1, 2010, includes positions for a total of 3414 extragalactic radio sources distributed over the entire sky. Such positions were determined from VLBI data acquired at 8.4 and $2.3 \mathrm{GHz}$ over 30 years (19792009). The ICRF2 has a floor of 40 microarcseconds ( $\mu$ as) in the individual source coordinate accuracies and an axis stability of $10 \mu$ as [1]. Joint observational efforts of the VLBI community aiming at a denser and even more accurate celestial frame continue in order to further improve the quality of the frame.

One limitation in improving the accuracy of the individual VLBI source positions originates in actual astrometric instabilities which are found in these positions. Due to their location at cosmological distances, no proper motion are expected for these sources and their astrometric positions should thus be stable with time. While this is indeed the case on the long term, it is not true for shorter time scales (months to years) where instabilities at the level of several hundreds of microarcseconds are commonly detected [1]. Such instabilities are usually attributed to varying source structure which is often spatially-extended at the level of the VLBI resolution [2]. Structural variations are generally due to ejection of material from the central VLBI core in a recurrent although unpredictable manner causing shifts in the brightness centroid of the radio emission and hence potential VLBI astrometric instabilities.

In this paper we compare source position instabilities and structural variations based on astrometric and imaging VLBI data covering a period of 10 years (1994-2003). The datasets are presented in Sect. 2 while Sect. 3 describes the analysis scheme. Section 4 reports our findings and discusses correlations between the two phenomena. In the last section, we draw prospects for further work in this area.

\section{Observations}

The data used to derive astrometric positions were acquired during numerous VLBI sessions conducted by the International VLBI Service for geodesy and astrometry (IVS) over the past 30 years [3]. A large number of observations is available for many sources which permits monitoring of their astrometric positions on time scales of days to weeks. Analysis was carried out in a similar way as that described in [4]. For our study we averaged the individual session-based positions over monthly intervals in order to have a sampling similar to that of the imaging data.

Structural variations were taken from the analysis of VLBI jet kinematics reported in [5]. This analysis has made use of 2753 images at $8.4 \mathrm{GHz}$ obtained from 50 Research \& Development VLBI sessions organized by the IVS and the Very Long Baseline Array between 1994 and 2003. Six such sessions are carried out every year with a sampling of approximately two months. The network includes 15-20 stations, yielding high-quality VLBI images. 68 sources observed at 20 epochs or more (with a median of 43 epochs per source) are included in the present study.

\section{Analysis}

For our study, we used a simplified representation of the source structures in the form of 


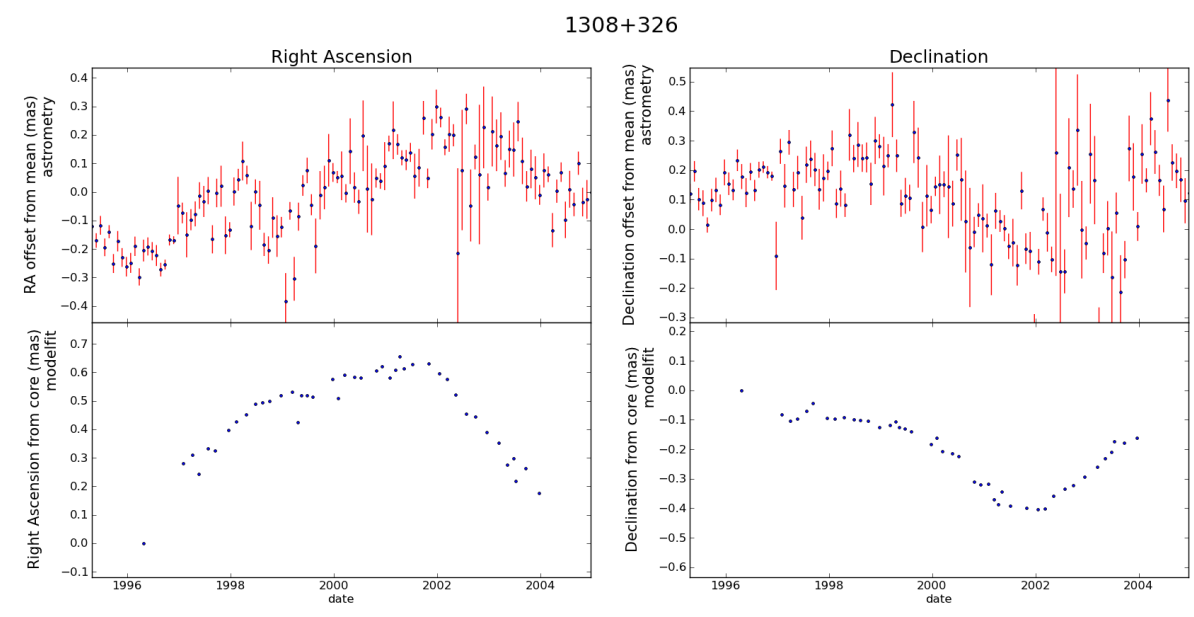

Figure 1: Comparison of the astrometric position instabilities (upper panels) and brightness centroid motions (lower panels) for the source 1308+326 between 1996 and 2003. The left panels are for right ascension while the right panels are for declination. The vertical scale is in milliarcseconds (mas).

a limited number of Gaussian components obtained through model-fitting (as available in [5]). Such a representation was preferred because it identifies the VLBI core of each source from epoch to epoch and aligns automatically the brightness distributions over time, assuming that the core position is stable. This alignment is crucial since the absolute map position is lost during the imaging process due to self-calibration.

Processing further the source structural information, we calculated the centroid of the brightness distribution (i.e. the centroid of the Gaussian components) at every epoch, allowing us to assess the relative motion of the brightness centroid with time. This calculation was carried out for 68 sources observed at 20 epochs or more for which model-fits are available in [5]. The result is a time series of centroid positions which may be compared with the monthly-averaged VLBI astrometric positions. This comparison assumes that the source motion as seen from the astrometric data is well matched with the motion of the centroid of the brightness distribution detected from VLBI imaging.

\section{Results and discussion}

An example of the comparisons that we carried out using the scheme explained in the previous section is presented in Fig. 1. The upper panels show the evolution in right ascension and declination of the astrometric position of the source $1308+326$ over 1996-2003, while the lower panels show the motion of its brightness centroid over the same period of time. Uncertainties in the astrometric positions were derived as weighted averages (over monthly intervals) of the individual session-based uncertainties. Declination has higher uncertainties due to the predominantly East-West baselines of current VLBI networks. No error bars are given for the centroid positions because model-fitting does not provide a direct mean to estimate reliable uncertainties for the Gaussian components representing the structures. 
Examination of the plots in Fig. 1 indicates similar trends in the evolution of the astrometric and brightness centroid positions. It is thus likely that astrometric instabilities for this source originate from structural variations. Similar correlations are also found for other sources in the sample although their visual significance is often not as large as that in Figure 1. Additionally, there are a number of sources for which it is not possible to draw any conclusions because there is no noticeable evolution in both the astrometric and structure-based positions. Finally, there are sources for which the two time series show apparent discrepancies. This includes the peculiar source 0923+392 (4C39.25) where such a discrepancy is understood (see [6] for a detailed investigation of this source). Others need a more thorough study on a case by case basis.

Different trends in the astrometrically-derived and structurally-derived positions may result from misidentification of the core component over the successive epochs (thereby affecting the alignment of images and the brightness centroid relative locations) or from effects coming from the $\mathrm{S}$ band $(2.3 \mathrm{GHz})$ data. For a full analysis, the $\mathrm{S}$ band structures should indeed be considered as well since the astrometric positions are derived from a combination of the data at the two frequencies whereas only the $\mathrm{X}$ band $(8.4 \mathrm{GHz})$ structures have been considered in the above comparisons. The $\mathrm{S}$ band data have a lower weight though and are thus a priori less likely to affect strongly the positions, hence the reason for neglecting them on first stage.

\section{Conclusions}

A comparison between the evolution of astrometric positions and the variations of source structure (characterized as the motion of the centroid of the brightness distribution) has been carried out for a sample of 68 sources observed over a period of 10 years between 1994 and 2003. This comparison reveals similar trends in the astrometric and structural time series of positions for some sources showing significant motions like $1308+326$. Such similarity favors an explanation of VLBI positions instabilities in terms of structural variations. On the other hand, the correlation for sources with smaller motions is more difficult to assess on a visual basis, while there are also cases where the astrometric and structural trends are different.

In order to evaluate the level of correlation in a more quantitative way, we plan to calculate correlation coefficients between the two time series (the astrometric and centroid positions) for all 68 sources in our sample. This should help to conclude about the degree of correlation between the two phenomena on a firmer base. Additionally, we plan to conduct detailed and specific studies for the sources which show different trends in the two time series in order to try understanding such unexpected behavior. Investigation of the magnitude of the effects caused by the $\mathrm{S}$ band data will also be carried out as these effects may affect our analysis at some level. In the longer term, enlarging the source sample and expanding the time span covered by the astrometric and imaging data up to recent years would be desirable.

\section{Acknowledgments}

The authors gratefully acknowledge support from the CNRS through the "Action Spécifique GRAM" for this project. 


\section{References}

[1] IERS \& IVS Working Group, The Second Realization of the International Celestial Reference Frame by Very Long Baseline Interferometry, IERS Technical Note 35, Eds. A. L. Fey, D. Gordon and C. S. Jacobs, Verlag des Bundesamsts für Kartographie und Geodäsie, Frankfurt am main (2009).

[2] A. L. Fey, \& P. Charlot, VLBA Observations of Radio Reference Frame Sources. III. Astrometric Suitability of an Additional 225 Sources., ApJS 128 (2000) 17-83.

[3] D. Behrend, Data Handling within the International VLBI Service., Data Science Journal 12 (2013) WDS81-WDS84.

[4] A.-M. Gontier, S. B. Lambert, \& C. Barache, The IVS team at the Paris Observatory: how are we doing?, in Proceedings of SF2A-2006: Semaine de l'Astrophysique Française (2006) 23.

[5] B. G. Piner, A. B. Pushkarev, Y. Y. Kovalev, C. J. Marvin, J. G. Arenson, P. Charlot, A. L. Fey, A. Collioud, \& P. A. Voitsik, Relativistic Jets in the Radio Reference Frame Image Database. II. Blazar Jet Accelerations from the First 10 Years of Data (1994-2003), ApJ 758 (2012) 84.

[6] A. L. Fey, T. M. Eubanks, \& K. A. Kingham, The proper motion of 4C39.25, AJ 114 (1997) 2284-2291. 